\title{
Using a mathematical model of cadherin-based adhesion to understand the function of the actin cytoskeleton
}

\author{
J. C. Dallon \\ dallon@math.byu.edu \\ Elijah Newren \\ Marc Hansen \\ Brigham Young University
}

Follow this and additional works at: https://scholarsarchive.byu.edu/facpub

Part of the Cell and Developmental Biology Commons, and the Mathematics Commons

Original Publication Citation

Physical Review E79(3): 031918 (2009)

\section{BYU ScholarsArchive Citation}

Dallon, J. C.; Newren, Elijah; and Hansen, Marc, "Using a mathematical model of cadherin-based adhesion to understand the function of the actin cytoskeleton" (2009). Faculty Publications. 2679.

https://scholarsarchive.byu.edu/facpub/2679

This Peer-Reviewed Article is brought to you for free and open access by BYU ScholarsArchive. It has been accepted for inclusion in Faculty Publications by an authorized administrator of BYU ScholarsArchive. For more information, please contact ellen_amatangelo@byu.edu. 
To appear in Physical Review E

\title{
Using a mathematical model of cadherin-based adhesion to understand the function of the actin cytoskeleton
}

\author{
J. C. Dallon \\ Department of Mathematics, Brigham Young University, TMCB 312, Provo, UT 84602-6539 \\ Elijah Newren \\ Sandia National Laboratories, Department 1541, Albuquerque, NM \\ Marc D. H. Hansen \\ Physiology and Developmental Biology, Brigham Young University, Provo, UT 84602-6539
}

(Dated: March 2, 2009)

\begin{abstract}
The actin cytoskeleton plays a role in cell-cell adhesion but its specific function is not clear. Actin might anchor cadherins or drive membrane protrusions in order to facilitate cell-cell adhesion. Using a mathematical model of the forces involved in cadherin-based adhesion we investigate its possible functions. The immersed boundary method is used to model the cell membrane and cortex with cadherin binding forces added as linear springs. The simulations indicate that cells in suspension can develop normal cell-cell contacts without actin-based cadherin anchoring or membrane protrusions. The cadherins can be fixed in the membrane or free to move and the end results are similar. For adherent cells, simulations suggest that the actin cytoskeleton must play an active role for the cells to establish cell-cell contact regions similar to those observed in vitro.
\end{abstract}

\section{INTRODUCTION}

Cadherin-based adhesion is critical in assembling individual cells into tissues. Initiation of cadherin-based adhesion between cells results in dramatic changes in actin organization [1-4]. Actin rearrangements include the formation of lamellae at or near the cell-cell contact, which have been proposed to expand the area of adhesion between individual cells $[5,6]$. Actin has also been thought to anchor engaged cadherin complexes, limiting their diffusion and providing a structural component that results in strong cell-cell adhesion [7]. Recent work reconstituting cadherin-actin complexes have called this assumption into question, however, as the proposed cadherin-catenin-actin complex cannot be reconstituted $[8,9]$.

A general role for actin in cell-cell adhesion is well accepted. Perturbations in the actin cytoskeleton with drugs do weaken cadherin-based adhesion between cells [10,11]. Similarly, perturbations of many actin regulatory systems result in altered cell-cell junction morphology and, not surprisingly, actin organization at cell-cell contacts [4, 12-16]. However, studies identifying players in actin regulation at cell-cell contacts do not address whether actin is required for direct cadherin anchoring or expansion and maintenance of the cell-cell contact area through actin-based membrane protrusions, or both. In fact, assessing these roles in isolation from one another is experimentally challenging. How can actin's function in protrusion formation be perturbed without altering other functions, such as cell-cell adhesion?

Here we use a mathematical model to examine the basic biophysical parameters that must exist for cells to initiate and expand their cell-cell junctions. The immersed boundary (IB) method can be used to model individual cells that display an elastic, deformable cortex. Cells coated with cadherins adhere to one another and will expand their junctions to a maximum area. Using this method, we independently test the roles of actin in cadherin anchoring or membrane protrusion formation in expansion of cell-cell contacts to a maximum cell-cell junction area.

Simulations using this model show that cadherin anchoring is not necessary to expand nascent cell-cell contacts. While membrane protrusions are not necessary to expand cell-cell junctions for cells in suspension, models which crudely mimic cells adhered to a substrate do not passively expand nascent cell-cell contacts fully, but do when membrane protrusions occur at the boundary of the cell-cell contact.

\section{DESCRIPTION OF THE MATHEMATICAL MODEL}

In our model we consider the forces exerted by cadherins, a cell's membrane along with its cortical shell, and the fluid surrounding the cells. We will divide our discussion of the model into two main areas - first, the representation of the cell membrane, cortical shell, and the fluid; second, the treatment of the cadherins. For the first part it is natural to use the IB method [17], which has been applied to a variety of biological fluid-structure interaction problems, including modeling of the cell with various degrees of complexity [18-20]. 


\section{A. IB method}

In the IB method, the coupled equations of motion for one or more elastic, massless surfaces immersed in a viscous, incompressible fluid are solved. The key idea of the IB method is to model the effect of the surface by a suitable force density term in the fluid dynamics equations; this allows a single set of fluid dynamics equations to hold in the entire domain with no internal boundary conditions. Thus the surface exerts forces on the fluid and the motion of the fluid determines the motion of the surface. The boundary is assumed to be massless, so that all of the force generated by distortions of the boundary is transmitted to the fluid. The boundary is modeled by a singular force, which is incorporated into the forcing term, $\mathbf{f}$, in the Navier-Stokes equations. The Navier-Stokes equations are then solved to determine the fluid velocity throughout the domain. Since the immersed boundary is in contact with the surrounding fluid, its velocity must be consistent with the no-slip boundary condition. Thus the immersed boundary moves at the local fluid velocity. Let $\Gamma$ represent the immersed boundary surface, $\mathbf{X}(s, t)$ be the parametrized curve in $\mathcal{R}^{2}$ that specifies points on $\Gamma, \mathbf{x}$ is a point in the domain $\Omega, \mathbf{u}$ is the fluid velocity, $p$ is the fluid pressure, and $t$ is time. The method is described by the following set of equations:

$$
\begin{aligned}
\mathbf{F}(s, t) & =\mathcal{A}[\mathbf{X}, t] \\
\mathbf{f}(\mathbf{x}, t) & =\int_{\Gamma} \mathbf{F}(s, t) \delta(\mathbf{x}-\mathbf{X}(s, t)) d s \\
\rho\left(\mathbf{u}_{t}+(\mathbf{u}) \cdot \nabla \mathbf{u}\right) & =-\nabla p+\mu \triangle \mathbf{u}+\mathbf{f} \\
\nabla \cdot \mathbf{u} & =0 \\
\frac{d \mathbf{X}(s, t)}{d t}=\mathbf{u}(\mathbf{X}(s, t), t) & =\int_{\Omega} \mathbf{u}(\mathbf{x}, t) \delta(\mathbf{x}-\mathbf{X}(s, t)) d \mathbf{x} .
\end{aligned}
$$

The operator, $\mathcal{A}=\mathcal{M}+\mathcal{C}$ has two parts: the first, $\mathcal{M}$, models the cell membrane and cortex assuming that it behaves like an object under elastic tension and the other part, $\mathcal{C}$, adds the force contributions due to binding cadherin pairs. The boundary, fluid force, and velocity are related through equations 2 and 5 . Equations 3 and 4 are the incompressible Navier-Stokes equations. We assume that the density, $\rho$, is constant and $\mu$ is the viscosity. Here $\delta$ is the Dirac delta distribution.

In our model there are two immersed boundaries which represent the membrane and cortical shell of two cells. The boundaries are discretized and represented by IB points. The cells are filled with and surrounded by water. Of course it would be more realistic to model the interior of the cell as a more complex material, but the model framework we are using only allows for one fluid. Due to this restriction, we include a section on model verification, where it is shown the simulations can reproduce experimental evidence in a different system with the same issue.

In our model the immersed boundary is a one dimensional representation of the two dimensional cell membrane. The elastic properties of a two dimensional network are more complex than those of a one dimensional network. In a one dimensional elastic spring, one elastic parameter will characterize the system, whereas in a two dimensional system a strain tensor describes the elastic properties of the system. Assuming certain symmetries the strain tensor for a two dimensional elastic membrane can be characterized by a shear modulus and an area compression modulus [21]. In our formulation the shear of the membrane is determined by a combination of the local stretching of the immersed boundary and the internal fluid pressure. We assume that for cells adhering in suspension, the membrane area of the cells does not change. Thus we use shear moduli for our model to determine the elastic properties of the immersed boundary. The stretching of the immersed boundary can be interpreted as the membrane becoming less flaccid locally.

The operator $\mathcal{M}$ is defined by assuming that the membrane and cortex behave like an object under elastic tension. For a boundary under tension, the strength of the force on the boundary is given by

$$
\mathbf{F}(s, t)=\frac{\partial}{\partial s}(T(s, t) \boldsymbol{\tau}(s, t))
$$

where $T(s, t)$ is the tension at the given point and $\boldsymbol{\tau}(s, t)$ is the tangent vector to the boundary at that point (see [22] for a derivation). The tangent vector is

$$
\boldsymbol{\tau}(s, t)=\frac{\partial \mathbf{X}}{\partial s} /\left\|\frac{\partial \mathbf{X}}{\partial s}\right\| .
$$

Assuming that the reference configuration represents an unstressed configuration, then $\left\|\frac{\partial \mathbf{X}}{\partial s}\right\|-1$ represents the strain. If we assume a Hooke's law material so that the force is proportional to the strain, then the tension is given by

$$
T(s, t)=T_{0}\left(\left\|\frac{\partial \mathbf{X}}{\partial s}\right\|-1\right) .
$$


If we instead assume that the boundary is linearly elastic with zero resting length (which we do not assume in this paper) then the tension becomes

$$
T(s, t)=T_{0}\left(\left\|\frac{\partial \mathbf{X}}{\partial s}\right\|\right)
$$

In the latter case $\mathcal{M}$ simplifies to $T_{0} \frac{\partial^{2}}{\partial s^{2}}$.

The discretized force operator is defined by

$$
\mathbf{F}_{k}^{n}=\mathbf{M}_{k}^{n}+\mathbf{A}_{k}^{n}
$$

where the superscript denotes the time and the subscript denotes the immersed boundary point (see the appendix for more details). The first part represents the membrane forces as an elastic substance which obeys Hooke's law. More specifically

$$
\mathbf{M}_{k}^{n}=\sum_{i} k_{M}\left(\left\|\mathbf{X}_{i}^{n}-\mathbf{X}_{k}^{n}\right\|-\ell_{M}\right) \frac{1}{\Delta s} \frac{\mathbf{X}_{i}^{n}-\mathbf{X}_{k}^{n}}{\left\|\mathbf{X}_{i}^{n}-\mathbf{X}_{k}^{n}\right\|}
$$

where the sum is over all IB points connected to $k, k_{M}$ is the spring constant for the membrane elasticity (for red blood cells $k_{M}=14 \mu \mathrm{N} / \mathrm{m}[23,24]$ and for MDCK cells $k_{M}=7$ dynes $\left./ \mathrm{cm}[25,26]\right), \ell_{M}=0.14 \mu \mathrm{m}$ is the rest length of the springs, and $\Delta s=0.156 \mu \mathrm{m}$ is the initial separation of the immersed boundary points. The initial separation of the immersed boundary points is set to be slightly higher than the spring rest length so the cell membrane will be under tension causing it to be circular in the absence of any other forces. Later in the manuscript we will describe the operator $\mathbf{A}_{k}^{n}$ which models the forces due to interacting cadherins.

\section{B. Numerical Implementation}

The Navier-Stokes equations are solved using a projection method, meaning that equation (3) is first solved with an approximation to the pressure gradient while ignoring the incompressibility constraint, and then a correction is performed which involves solving a Poisson equation in order to enforce the incompressibility constraint and obtain a more accurate pressure approximation.

Due to the parameters in our system, $\rho=1 \mathrm{~g} / \mathrm{cm}^{3}, \mu=0.01 \mathrm{~g}$ per sec per $\mathrm{cm}$, and the characteristic length being the order of microns, we can approximate the solution with a Stokes flow, i.e, we replace equation (3) with

$$
0=-\nabla p+\bar{\mu} \triangle \mathbf{u}+\mathbf{f}
$$

where the variables have all been scaled appropriately. This simplification also allows the Navier-Stokes solver to be replaced with three Poisson solves (one for the pressure, followed by one for each of the velocity components).

A comparison of numerical solutions of Stokes flow, $U_{S}\left(k_{1}\right)$ and $U_{S}\left(k_{2}\right)$ calculated using two time steps $10 k_{1}=k_{2}$, with solutions of the full Navier-Stokes equation, $U_{N}\left(k_{1}\right)$ and $U_{N}\left(k_{2}\right)$, verified that Stokes flow is a good approximation. If we assumed that $U_{N}\left(k_{1}\right)$ is accurate, we found that $U_{S}\left(k_{2}\right)$ is a better approximation than $U_{N}\left(k_{2}\right)$, and $U_{S}\left(k_{1}\right)$ was almost identical to $U_{N}\left(k_{1}\right)$. The theoretical analysis indicates that Stokes flow should be a good approximation and the numerical results are consistent with this prediction. All the simulations shown in the paper are solved using Stokes flow.

We have implemented a slightly modified interpolation scheme in our Immersed Boundary implementation. The interpolated velocity field specified by equation 5 will not in general maintain the incompressibility of the fluid, which can result in an observable volume loss during the course of the simulation. We addressed this problem by introducing a correction to the interpolated velocity field which ensures that the corrected velocity field satisfies a discrete incompressibility condition. More details on the correction can be found in [27] and the appendix.

\section{Cadherins}

The cadherins are modeled as discrete complexes on the cell membrane (the immersed boundary). A complex can represent more than one cadherin and more than one complex can occupy the same location or site. The number of caherins in a complex is the cadherin weight. Cadherin complexes interact with cadherins complexes on the other cell and they can move within the cell membrane. The locations of the cadherin complexes or the sites of the cadherin complexes are constrained to the IB points which define the membrane, but cadherin complexes can move from point to point and can accumulate at points. From now on in the paper we will refer to a cadherin complex as simply a cadherin. 


\section{Cadherin motion}

The cadherins can diffuse in the membrane and move due to sustained directional forces (convective motion). The diffusive motion of the cadherins is modeled by a random walk with the diffusion coefficient for an individual cadherin taken to be $1 \times 10^{-10} \mathrm{~cm}^{2} / \mathrm{s}$ [28]. If the diffusive motion of a pair of bound cadherins would cause them to separate and break the bonds, the cadherins do not move. In the results section we discuss how varying the diffusion coefficient affects the system.

Any forces on the cadherin can cause a convective motion within the membrane. We will assume that motion in the membrane occurs at low Reynold's number and can be approximated by

$$
\mathbf{F}=\gamma \mathbf{v}
$$

where $\mathbf{F}$ is a force vector, $\gamma$ is the drag coefficient, and $\mathbf{v}$ is the velocity of the cadherin. Of course, the motion is constrained so that the cadherin remains in the cell membrane. We determine $\gamma$ from the Einstein relationship

$$
D=\mu k T
$$

where $D$ is the diffusion coefficient, $k$ is Boltzmann's constant, $T$ is the temperature (assumed to be 37 degrees Celsius), and $\mu$ is the mobility. At low Reynolds number, $\mu=\frac{1}{\gamma}$. The motion is determined in the model by projecting the forces on the cadherin onto a unit vector tangent to the cell membrane at the position of the cadherin and averaging this force for the two linked cadherins. Using equation (12) we determine the velocity of the cadherin. We use Euler's method to determine the distance the cadherin should move. If it is greater than half the distance to the next IB point, the cadherin moves. This approximation is for simplicity and should result in making the cadherins less mobile than normal. The distance between the IB points in the model is variable but for simplicity we assume it is constant and take the value to be the separation given in the initial conditions.

\section{Cadherin adhesions}

We assume that a cadherin binds to only one cadherin on the other cell. When a cadherin from one cell gets close enough to a cadherin on the other cell they will interact and bind to one another which means they will exert forces on each other. The interaction distance is taken to be $40 \mathrm{~nm}$ [29] and the interaction force attains a maximum of 35 pN per individual cadherin molecule when the cadherin sites are $40 \mathrm{~nm}$ apart (and the membranes have not crossed over each other) [29]. The force is modeled as a linear spring when stretched and the discretized operator $\mathbf{A}_{k}^{n}$ is given by,

$$
\mathbf{A}_{k}^{n}= \begin{cases}w k_{c}\left(\left\|\mathbf{X}_{a}^{n}-\mathbf{X}_{k}^{n}\right\|-\ell_{c}\right) \frac{1}{\Delta s} \frac{\mathbf{X}_{a}^{n}-\mathbf{X}_{k}^{n}}{\left\|\mathbf{X}_{a}^{n}-\mathbf{X}_{k}^{n}\right\|} & \text { if cadherin is bound } \\ 0 & \text { otherwise }\end{cases}
$$

where $w$ is the weight, $k_{c}=1.75$ dynes $/ \mathrm{cm}, \ell_{c}=20 \mathrm{~nm}, \mathbf{X}_{k}^{n}$ is the location of the current cadherin, and $\mathbf{X}_{a}^{n}$ is the location of the cadherin to which it is adhering. If the cell membrane overlaps or if the cadherins become separated by more than 40nm the cadherins do not exert force.

\section{MODEL VERIFICATION}

Although our model treats the forces involved in cell-cell adhesion in a more complex manner than most previous models, the formulation of the cell membrane and cytoplasm is simplistic. We use red blood cells to test the validity of our model because their membrane dynamics and properties have been widely studied [30]. Though red blood cells and MDCK cells are vastly different from each other, their membrane and cytoplasmic properties are equally different from the model assumptions. The two model assumptions we are referring to are making the cell membrane and the cortical shell one elastic structure and assuming the cytoplasm has the viscosity of water.

We simulated experiments where a red blood cell is deformed using optical tweezers [23]. For red blood cells the spring constant for the membrane elasticity in our model is $k_{M}=14 \mu \mathrm{N} / \mathrm{m}[23,24]$. When a force of $340 \times 10^{-12}$ newtons was applied to the cell, it stretched 50 percent of its diameter in the direction of the applied force and the diameter transverse to the stretching direction was decreased by 40 percent [23, 24]. In those studies, the authors calculated shear moduli of 22.5 microNewton per meter for low shear strains and 13.3 microNewton per meter for high shear strain from their theoretical model which match the experimental data. The stretching occurred in 2-5 
seconds. They observe that a simple spherical model was not able to match the change in diameters in both directions. Additionally they noted that their membrane shear moduli values are larger than those measured using micropipette aspiration experiments [31].

Our simulations matched experimental data using reasonable parameters. We simulated a cell of diameter 10 microns with a force of $340 \times 10^{-12}$ newtons applied to each side using a shear modulus of 14 microNewton per meter (see figure 1). Within six seconds the cell had almost reached a steady-state; diameters were elongated by

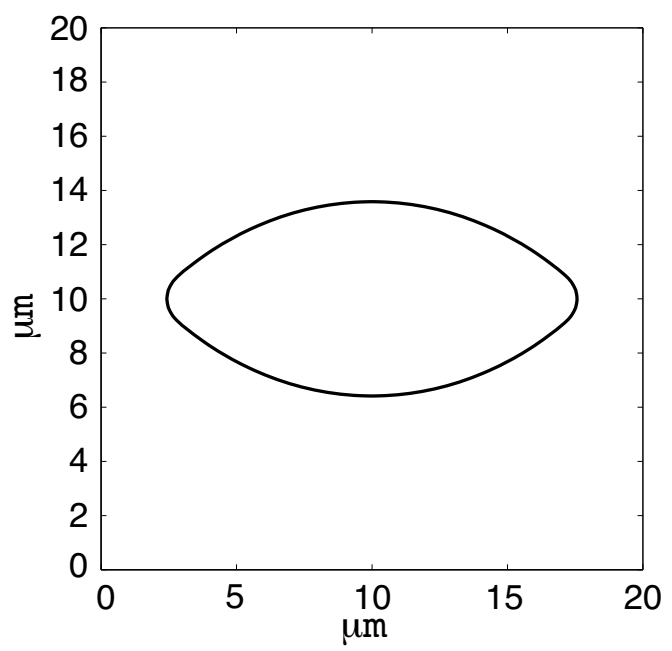

FIG. 1: Results of a simulation designed to mimic experiments where red blood cells are stretched. The membrane is initially a circle with a ten micron diameter. The cell shape is shown two seconds after a total force of $340 \times 10^{-12}$ newtons is applied to a two micron arc on both the right and left side of the cell membrane. Over 99 percent of the elongation occurred within two seconds and steady state was basically reached within six seconds. The time step in the explicit scheme is fixed at $1 \times 10^{-5}$ seconds.

52.4 percent parallel to the direction of the force and reduced 29.3 percent in the transverse direction. Most of the elongation occurred in the first two seconds. Although our model formulation is very different from that used in the experimental work, it mimics the elongation of the cell in the correct time frame with similar parameter values. This gives confidence that by changing the parameters of membrane elasticity from those measured for red blood cells to those measured for MDCK cells, we can accurately model MDCK cells during cell-cell contact formation. The appropriate spring constant for the membrane elasticity in our model for MDCK cells is $k_{M}=7$ dynes/cm $[25,26]$.

\section{RESULTS}

We now describe the results of the computational simulations. For simplicity, the simulations model two MDCK cells in suspension adhering to each other, and interactions with a substrate are not considered initially. In order to understand the effects of cadherin motion within the membrane on the length of cell-cell interaction, the first set of simulations has cadherin which are fixed in the membrane. This is intended to mimic the ability of the cytoskeleton to anchor the cadherins and restrict their movement in the membrane. Motion within the membrane is added in later simulations by first considering simple lateral diffusion, then convection of the cadherins, and finally both diffusion and convection. In this manner we can compare the differences that would occur if the cell actively restricts the motion of cadherins in the membrane or if the cadherins can freely move.

\section{A. Cell-cell junctions in suspension}

In order to determine the cellular morphology of two cells adhering in suspension, MDCK (Madin-Darby canine kidney) cells were placed in suspension culture at 250,000 cells $/ \mathrm{ml}$ in a $20 \mu \mathrm{l}$ drop that was suspended from the lid of a petri dish. Cells were allowed to adhere to one another for 30 minutes before the drop was spread on a microscope slide and images of paired cells recorded (figure 2a). The length of a cell-cell junction, relative to the diameter of each cell in a pair was determined for 21 adherent pairs using ImageJ [32]. The average ratio of the length of the contact area of the cell-cell interaction and the cell diameter in the experiments is 0.60 with a standard deviation of 0.10. 


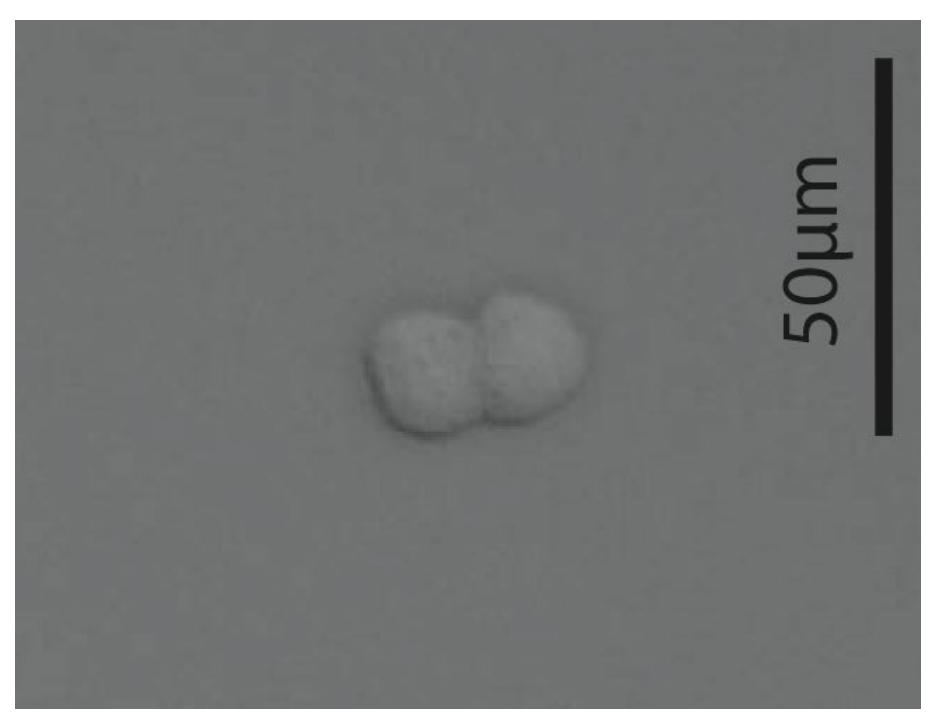

a

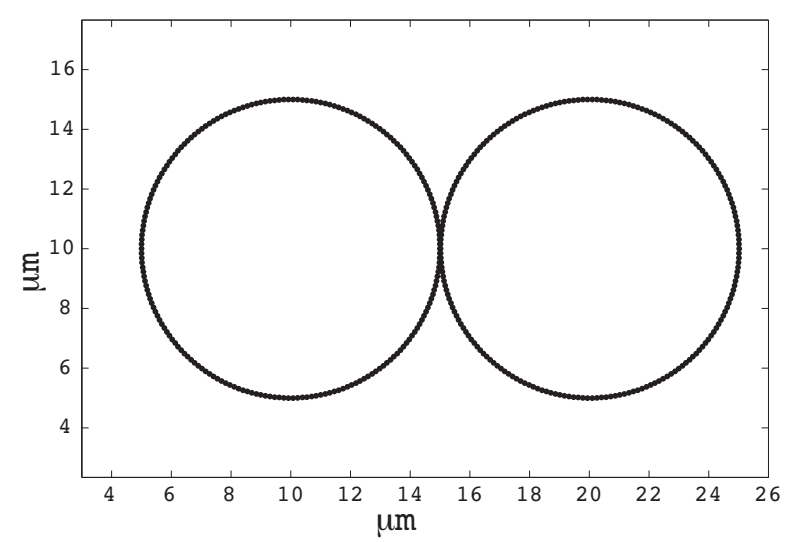

$\mathrm{b}: t=0$ seconds

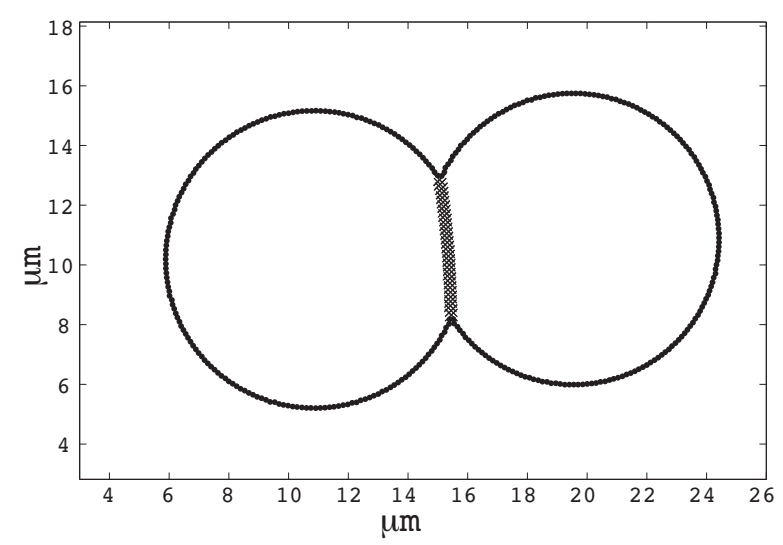

c: $t=60$ seconds

FIG. 2: This figure shows in vitro cells adhering and results of a mathematical simulation of cells adhering. Part a) shows a pair of MDCK cells in suspension culture with a cadherin based junction. In b) the initial conditions of a typical simulation of adhering MDCK cells is shown and in c) the cells' configuration after 60 seconds of simulated time is shown. In this simulation the cadherins are fixed in space and each cadherin represents 100 individual cadherin molecules. The cadherin which are bound are denoted by an "X" and the cadherin which are not bound are black dots.

\section{Stationary cadherin}

In the first set of simulations the cadherin are fixed in the membrane and not allowed to diffuse laterally. These simulations are designed to test the effects of a cells actively anchoring the cadherins in the membrane, preventing them from moving. For this model, figure $2 \mathrm{~b}$ shows the initial conditions and figure 2c shows a typical simulation after 60 seconds.

The measurements of the stable contact length relative to cell diameter in our model compare well with values determined for MDCK cells in suspension culture. The length of the nascent cell-cell contact as a function of time is shown in figure 3a, which demonstrates how the rate of contact expansion is initially rapid and reaches a plateau phase as it approaches the maximal contact length.

In order to examine how increasing the number of cadherins at the membrane might alter contact expansion, we increased the number of cadherin molecules for each cadherin complex on the membrane, the cadherin weight. Although cells with an increased number of individual cadherin molecules per complex reached a steady state contact length earlier, the final contact length was largely unaffected (see figure 3a). 


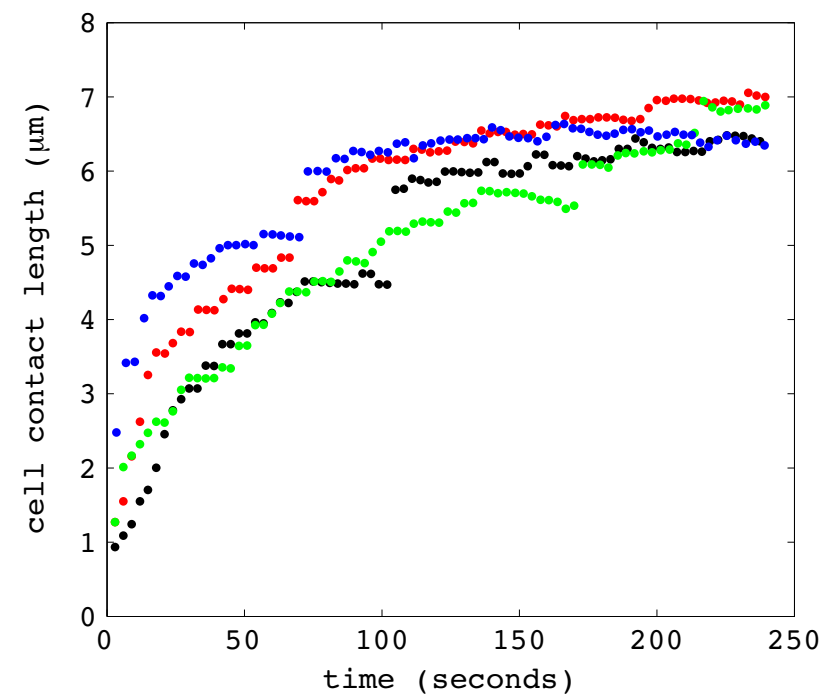

a

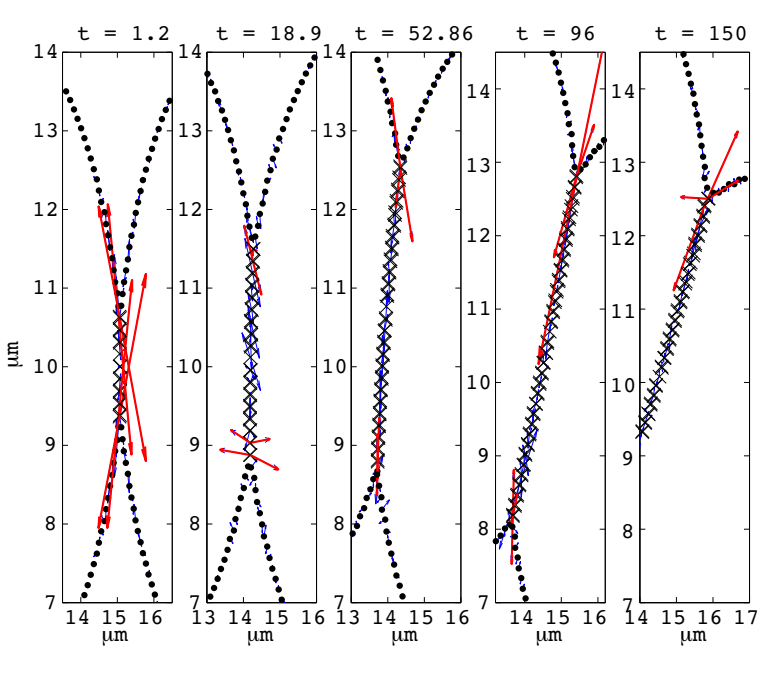

$\mathrm{b}$

FIG. 3: (Color online) Simulation results where the weight of the cadherins are varied showing the length of the MDCK cell contact over time. Part a) is a graph of the contact length as it changes with time. Simulations with 5 individual cadherin molecules per complex are shown in black, 50 individual cadherin molecules per complex are shown in red (medium gray), 100 individual cadherin molecules per complex are shown in green (light gray), and 500 individual cadherin molecules per complex are shown in blue (dark gray). In part b), high magnification images of the interface between bound membranes and unbound membranes in frames from a simulation of laterally fixed cadherins with 100 individual cadherin molecules per complex. Arrows represent the force vectors on the membrane. The two bound cadherin adjacent to unbound cadherins have the associated force vectors colored red and the rest are blue. Notice how forces are greatest near the interface, but stabilize. In b) the cadherins denoted by "X" are bound and cadherins denoted by black dots are unbound. For these simulations there is no diffusion and no convection.

\section{Cadherins with lateral diffusion}

In order to assess how anchoring of cadherins contributes to rapid cell-cell junction expansion, we ran a set of simulations where cadherins were free to diffuse in the plane of the membrane. All other parameters from simulations of fixed cadherins were retained. Free lateral mobility of cadherins did not affect the long term contact length but did result in an altered rate of cell-cell contact expansion. This seems to be true for a large range of values for the diffusion coefficient. Surprisingly, the initial rate of contact expansion is faster, as well as more saltatory (see figure 4). The length of the contact at plateau phase is between 0.6 and 0.7 of a cell diameter, very similar to that of actual cells. We observe that diffusible cadherins tend to cluster towards the boundary between the contacting and free membrane surfaces, resulting in a significantly reduced level of cadherin density on the membrane at the center of the contact and, particularly, just outside the boundary of the contact (figure 5). This is natural since in the model it is assumed that the force of diffusion does not break the cadherin-cadherin bonds in this simulation. The break in the contact between membranes acts like a barrier to diffusion until the membranes move close enough for the cadherins to interact resulting in the accumulation at that boundary. The low density region results in an early slowing of contact expansion as abutted membranes devoid of cadherins fail to adhere later in the simulation. This situation could be overcome with local deposition of newly synthesized or recycled cadherins.

\section{Influence of convective forces on cadherins}

We reasoned that a lack of convective forces in our model involving mobile cadherins might give us unanticipated results. In order to address this we examined how such forces altered cell-cell contact expansion. The convective forces depend on the diffusion coefficient via the Einstein relationship as stated previously. When convective forces were first added to simulations with no lateral diffusion using a realistic diffusion coefficient of $1 \times 10^{-10} \mathrm{~cm}^{2} / \mathrm{s}$, the initial contact expansion was more rapid than with fixed cadherins, but not much different from diffusing cadherins without convective forces (see figure $4 \mathrm{~b}$ ). The overall impact of allowing the cadherins to move within the membrane does not affect the final length of the contact region. 



$\mathrm{b}$

FIG. 4: (Color online) Simulation results showing the change in length of the MDCK cell contact with time when cadherins are allowed to move. In a) the cadherins laterally diffuse in the membrane at different rates resulting in little overall effect on the final contact length. For the simulations shown in black the diffusion constant is 0 , for red (medium gray) it is $1 \times 10^{-12}$, for green (light gray) it is $1 \times 10^{-10}$ (a realistic value), and for blue (dark gray or the top line) it is $1 \times 10^{-6}$. In b) simulations with fixed cadherins are shown in black, cadherins which diffuse are shown in red (medium gray), cadherins which convect are shown in green (light gray), and cadherins which both diffuse and convect are shown in blue (dark gray). In b) the diffusion rate is fixed at $1 \times 10^{-10} \mathrm{~cm}^{2} /$ sec. In these simulations the cadherin weight is 100 . The simulation with the largest diffusion coefficient ended prematurely due to computational difficulties.

Interestingly, if the diffusion coefficient is higher, $7 \times 10^{-8} \mathrm{~cm}^{2} / \mathrm{s}$, allowing convection makes a more dramatic difference. In the set of simulations shown in figure 6 , adding convection increases the rate at which the contact region grows when compared to simulations with fixed or freely diffusible cadherins without convective forces applied. This seems to result from movement of bound cadherins at the edge of the contact area. Bound cadherins at the interface between the junction and the free surface exert larger forces on the cell cortex. As these bound cadherins move away from and then back towards the interface, a result of convective forces, forces on the cell cortex are relaxed and then suddenly reimposed at the interface. The result is a membrane fluctuation that is driven past the interface and onto the cell cortex of the free surface, causing in the free membrane of apposed cells to be drawn together more quickly (see figure 6b). Drawing apposed membranes into closer proximity in this manner increases the likelihood of cadherin-cadherin interactions occurring at areas adjacent to the edge of the cell-cell contact. At plateau phase, the contact length of the simulation with convection only was comparable to that for models without convective forces.

We then tested at the higher diffusion rate, whether addition of convective forces would reduce the ability of cells with cadherins which laterally diffuse to expand cell-cell adhesions. Surprisingly, adding both types of cadherin motion, random diffusion and motion due to convective forces, resulted in the contact length growing to a larger value than in other simulations (see figure 6a). The rate of expansion was both more rapid and more saltatory. The likely reason is that engaged cadherins pairs that exert large forces at the contacting and free membrane interface are more free to move laterally. The result is that the loss and re-imposition of large forces at the interface is more pronounced, driving larger membrane fluctuations through to the free membrane surface and bringing the free membranes near the interface of apposing cells together much more rapidly. A low density of cadherins is observed in this region later in the simulation.

\section{B. Simulations with high viscosity that model cell-substrate adhesion}

We then wondered how a model of cells adhering in suspension conditions could be compared to those making contact while already adhering to a substrate. A modification of this model to consider a cell's interaction with a substrate is to increase the viscosity of the system [33-35]. The cell interacts with the substrate via integrins, which bind and unbind to the substrate. In a fluid, interaction between the molecules is modeled by viscosity. Increasing the viscosity represents increasing the strength of interaction between the molecules. Increasing the viscosity does not 


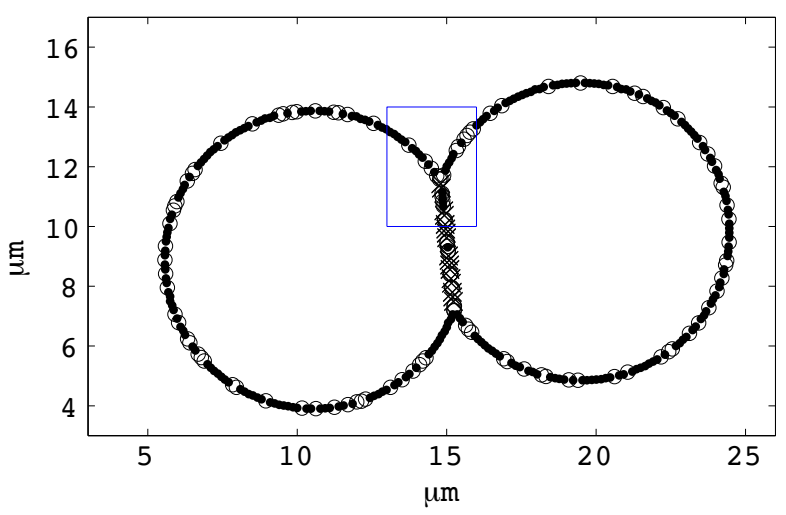

a



b: blowup

FIG. 5: In this image the low density of cadherins near the cell-cell interaction region can be seen in a simulation with laterally diffusing cadherins. The image in b) is a blow up of the boxed region in a). The simulation has cadherin complexes weighted by 100. The time shown is 30 seconds into the simulation. The cadherins which are bound are denoted by an "X", the cadherins which are not bound are black dots, and the sites which are empty are denoted with an open circle.

directly increase the interacting forces between the immersed boundary (the cell membrane) and the fluid (substrate), but does increase the force required to move the boundary in the fluid. Integrin binding and unbinding increases the force required for the cell to move not unlike an increase in the forces of interacting molecules requires more force for an object to move through a fluid. In simulations where the viscosity of the fluid is increased by a factor of $10^{4}$ the region of contact between the two membranes did not grow beyond 2 microns within 400 hours with or without cadherin diffusion and convection.

We sought to examine how the cell overcomes this problem by focusing on the role of actin-based protrusions. Cells on substrates demonstrate increased membrane protrusive activity that is focused towards nascent cell-cell junctions [6]. The stall forces of lamellipodia in keratocytes has been measured as $2 \mathrm{nN}$ per $\mu m^{2}$ [36], and in our model a force of $0.2 \mathrm{nN}$ per $\mu \mathrm{m}^{2}$ causes a protrusion of about 5 microns in length in about 48 seconds. To mimic forces resulting from membrane protrusions, a radial force of magnitude ranging from 0.02 to $0.2 \mathrm{nN} \mu \mathrm{m}^{2}$ representing pseudopodial activity was applied to the cell membrane on an arc of 1.4 microns away from the interface between the contacting and free membranes of each cell. In simulations with stationary cadherins weighted at 100 and where pseudopod forces are applied, the final contact area increased to about 10 microns in 30 minutes (see figure 7) as the force of the pseudopod increased. Simulations with freely diffusible cadherins failed because pseudopods forced together apposed membranes devoid of cadherins and, without adhesion, the membranes moved through each other and into the adjacent cell. Taken together, these results suggest that pseudopod activity is necessary to obtain the observed contact length of cells on a substrate. The pseudopod extension allows membranes to be forced into apposition long enough for cadherins from opposite cells to bind and stabilize the membrane contact.

\section{DISCUSSION}

Traditional understanding of cadherin-based adhesion is founded on cadherin anchoring by actin filaments. Anchoring is thought to restrict cadherin mobility and allow clustering of cadherins on the cell surface. Recent evidence indicates that the cadherin complex does not directly associate with actin filaments [9]. Instead, cadherin complexes may direct changes in local actin organization [8]. It remains unclear how unanchored cadherin molecules with full lateral mobility in the membrane would be able to drive expansion of cell-cell contacts. Here we present the results of a mathematical model of cell-cell junction expansion in cells with variable cadherin mobility. While our initial hypothesis was that full cadherin mobility would limit expansion of cell-cell contacts beyond a single point of adhesion, results show that full lateral mobility of cadherin complexes allow expansion of the cell-cell junction. Application of convection forces at the boundaries between cell-cell contacts and free membrane surfaces does not reduce contact 


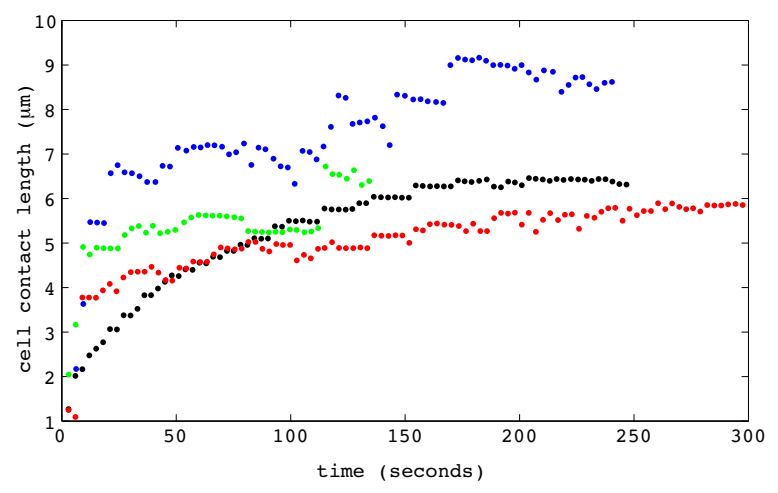

a

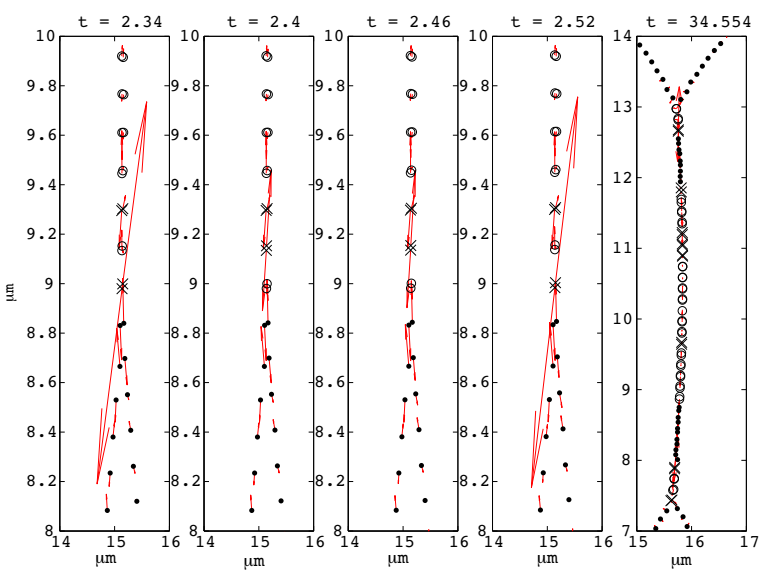

$\mathrm{b}$

FIG. 6: (Color online) Simulation results showing the change in length of the MDCK cell contact with time when cadherins are allowed to move in the membrane with a diffusion constant of $7 \times 10^{-8}$. It is interesting that at higher diffusion rates the convection has a greater impact on the contact length. In part a) simulations with fixed cadherins are shown in black, cadherins which diffuse are shown in red (medium gray), cadherins which convect are shown in green (light gray), and cadherins which both diffuse and convect are shown in blue (the highest line). In part b) high magnification images of the interface between bound membranes and unbound membranes in frames from a simulation with lateral cadherin motion due to convective forces only is shown. At $t=2.4$ seconds the bound cadherin pair has moved relaxing the forces at their previous location. At $t=2.46$ the cadherin pair has moved back and the forces are reapplied. This motion causes more fluctuations in the membrane and a longer contact area sooner than in the case where the cadherins do not move or move with diffusion only. In these simulations the cadherin weight is 100 . In part b) the simulation has convective forces acting on cadherins but no diffusion. The red arrows represent the force vectors on the membrane. The cadherins denoted by " $\mathrm{X}$ " are bound, cadherins denoted by black dots are unbound, and open circles denote IB points with no cadherins.

expansion. Surprisingly, fixing cadherins by anchoring to actin filaments, as proposed in traditional understanding of cell-cell adhesions, does not appear to be necessary for establishment of cell-cell junctions. What, then, might be the role of actin in cell-cell adhesion? Cells clearly demonstrate altered actin organization at cell-cell contacts, and changes in actin dynamics are apparent as epithelial cells initiate and establish cell-cell contacts [4, 37]. Increased membrane protrusions are observed at cell-cell contact sites, and have been proposed in facilitating rapid expansion of the cell-cell contact [6]. Our model, however, best represents cell-cell junction assembly between cells in suspension, where limited membrane protrusion opportunities are likely to occur. In suspension, epithelial cells do adhere and expand their junctions, though the maximum contact length, as a function of cell diameter, is reduced compared to substrate adherent cells. In our suspension model, contact expansion rapidly reaches a plateau phase, a point beyond which active membrane protrusion may be necessary. Models of cell contact expansion in high viscosity medium supports this idea. In such simulations contact expansion is severely limited no matter how cadherin's lateral diffusion properties are varied nor whether convective forces are present. However, addition of protrusive forces at the boundaries of the cell-cell contact, mimicking those observed in real cells [6], allows rapid and complete contact expansion. The results of this model indicates that a primary role of actin in cell-cell contact formation may be expansion of the contact length by formation of membrane protrusions.

Analysis of forces exerted on cadherin complexes reveal that large forces appear in waves and usually at the boundary between the contacting and free membranes of the cell-cell contact. Forces along the length of the contacts are much smaller. This is particularly apparent when convective forces are added, which move cadherins in and out of the boundary between the contacting and free membrane surfaces. The result is large force fluctuations at the interface that drive adjacent free membranes from apposed cells together. These "forced" membrane protrusions increase the rate of cell-cell contact expansion, particularly for cells with freely diffusible cadherins. A role for membrane protrusion is further supported by our simulations of cells in a high viscosity conditions designed to mimic substrate adhesion, where contact expansion is severely limited. While neither convective forces nor cadherin mobility allow increased contact expansion, addition of membrane protrusive forces had a significant effect, driving free membranes of contacting cells together and increasing both the rate of contact expansion and the maximal contact length. These results indicate that focusing membrane protrusions towards nascent cell-cell contacts could be critical in establishment of cell-cell adhesions for substrate adherent cells.

Interestingly, the interface between the contacting and free membrane surfaces, the site of large force fluctuations, 


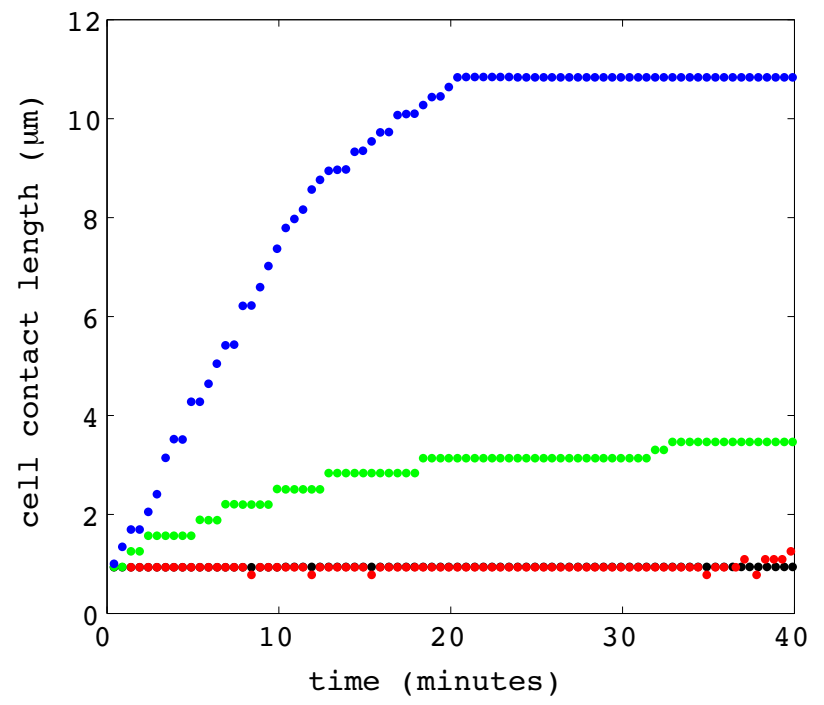

a

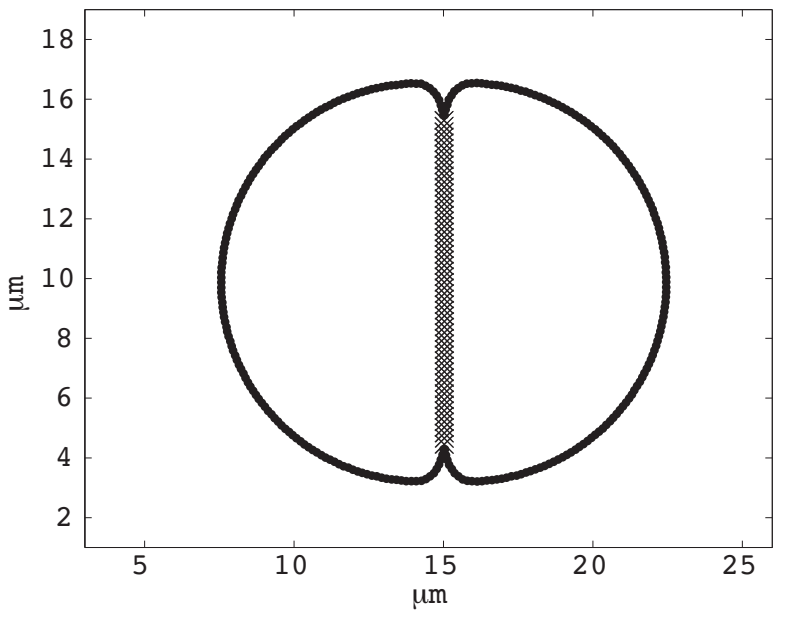

$\mathrm{b}$

FIG. 7: (Color online) This image shows results from simulations for MDCK cells on substrate. In a) the length of the contact region is plotted in time for simulations with cadherins which are fixed in the membrane shown in black (the lowest line of dots), cadherins which laterally diffuse and move with convective forces shown in red (medium gray, mostly superimposed on the black), fixed cadherins and pseudopods exerting a force of $0.02 \mathrm{nN}$ per $\mu \mathrm{m}^{2}$ shown in green (light gray), and fixed cadherins and pseudopods exerting a force of $0.2 \mathrm{nN}$ per $\mu \mathrm{m}^{2}$ shown in blue (the highest line of dots). In b) the cell membranes are shown for the simulation shown in blue in a) at 30 minutes.

shows dramatically different actin organization from the rest of the cell-cell contact in real cells. A thin line of actin is observed at the length of cell-cell contacts, while a thick cable of filaments abuts the membrane at a near perpendicular angle at the ends of the contact [2]. Such different organizations may be critical in handling forces exerted during contact expansion. Zyxin and VASP are localized to the ends of such thick cables [4, 16], perhaps serving to link actin filaments to membranes at these sites. Further, zyxin and VASP have been recently implicated in remodeling actin in response to stretch forces at actin cables that emanate from focal adhesions [38]. Actin organization at cell-cell junctions could be altered as a direct result of forces applied on points of the cell-cell contact.

Here we directly address the role of cadherin anchoring in establishment and expansion of cell-cell adhesions. Surprisingly, cadherin mobility is not a factor in the majority of cell-cell junction expansion. These results provide additional evidence that traditional thinking about how actin contributes to cell-cell adhesion must be reevaluated.

\section{APPENDIX}

\section{A. Numerical details}

The most common temporal discretization of the equations describing the IB (immersed boundary) method is a mixed explicit/implicit one, where the forces from the immersed boundary and the advection terms in the NavierStokes equations are treated explicitly while the viscous terms are treated implicitly. The work involved in a timestep of such a method consists of five steps: (1) calculation of forces on the immersed boundary, (2) spreading forces from the immersed boundary to the Eulerian grid, (3) solving the Navier-Stokes equations with the resulting Eulerian force, (4) interpolating velocities from the Eulerian grid to the immersed boundary, and (5) updating immersed boundary points according to the interpolated velocity.

We use a superscript to denote the value of a variable at a given timestep; thus $\mathbf{u}^{n}(\mathbf{x})=\mathbf{u}(\mathbf{x}, n \Delta t)$ and $\mathbf{X}^{n}(s)=$ $\mathbf{X}(s, n \Delta t)$. Using this notation, temporally discretizing equations (1) through (5) according to the steps listed above using an explicit (forward Euler) handling of all immersed boundary and an implicit (Crank Nicholson) discretization 




FIG. 8: Example discrete immersed boundary curve and underlying discretized Eulerian grid

of the viscous terms, we obtain

$$
\begin{gathered}
\mathbf{F}^{n}(s)=\mathcal{A}_{f} \mathbf{X}^{n}(s) \\
\mathbf{f}^{n}(\mathbf{x})=\int_{\Gamma} \mathbf{F}^{n}(s) \delta\left(\mathbf{x}-\mathbf{X}^{n}(s)\right) \mathrm{d} s \\
\rho \frac{\mathbf{u}^{n+1}-\mathbf{u}^{n}}{\Delta t}=-\nabla p^{n+\frac{1}{2}}-\rho[(\mathbf{u} \cdot \nabla) \mathbf{u}]^{n+\frac{1}{2}}+\frac{\mu}{2} \Delta\left(\mathbf{u}^{n+1}+\mathbf{u}^{n}\right)+\mathbf{f}^{n} \\
\nabla \cdot \mathbf{u}^{n+1}=0 \\
\frac{\mathbf{X}^{n+1}-\mathbf{X}^{n}}{\Delta t}=\mathbf{u}^{n+1}\left(\mathbf{X}^{n}(s)\right)=\int_{\Omega} \mathbf{u}^{n+1}(\mathbf{x}) \delta\left(\mathbf{x}-\mathbf{X}^{n}(s)\right) \mathrm{d} \mathbf{x} .
\end{gathered}
$$

These equations are solved on a pair of computational grids: a Cartesian grid for Eulerian variables, and a discrete set of points for the Lagrangian variables. An example setup in 2 dimensions with a single immersed boundary curve on a very coarse mesh is shown in Figure 8.

Assuming that the lower left corner of the domain is at the origin, the coordinates of the $i j$-th Eulerian gridpoint are $\mathbf{x}_{i j}=\left(\left(i+\frac{1}{2}\right) \Delta x,\left(j+\frac{1}{2}\right) \Delta y\right)$. A pair of subscripts on a variable denotes the location at which the Eulerian variable is being evaluated; thus $\mathbf{u}_{i j}$ denotes the value of the variable $\mathbf{u}$ at the $i j$-th gridpoint. Lagrangian gridpoints are identified by a single index, with variables at such gridpoints identified by the corresponding index appearing as a subscript. Thus $\mathbf{F}_{k}$ denotes the value of the variable $\mathbf{F}$ at the $k$-th gridpoint. The location of the $k$ th Lagrangian gridpoint is explicitly tracked in $\mathbf{X}_{k}(t)$. The value $\Delta s$ is typically taken to be the initial separation of the immersed boundary points if they are evenly spaced.

The interaction between these grids, governed by integration against a delta function in the spatially continuous equations (14) and (17), is handled by introducing a regularized discrete delta function whose support is comparable to the mesh spacing. The spatially discretized forms of equations (14) and (17) using this discrete delta function are

$$
\begin{gathered}
\mathbf{f}_{i j}^{n}=\sum_{k} \mathbf{F}_{k}^{n} \delta_{h}\left(\mathbf{x}_{i j}-\mathbf{X}_{k}^{n}\right) \Delta s \\
\frac{\mathbf{X}_{k}^{n+1}-\mathbf{X}_{k}^{n}}{\Delta t}=\mathbf{U}_{k}^{n+\frac{1}{2}}=\sum_{i j} \mathbf{u}_{i j}^{n} \delta_{h}\left(\mathbf{x}_{i j}-\mathbf{X}_{k}^{n}\right) \Delta x \Delta y
\end{gathered}
$$

The discrete delta function appearing in equations (18) and (19) is derived from the requirement that a certain set of properties be satisfied; these include, ensuring that the entire force is transmitted to the grid, that the force 
density on the grid is a continuous function of the IB point locations, and that the communication between Eulerian and Lagrangian grids is very localized. We will use the delta function derived in [39],

$$
\begin{gathered}
\delta_{h}(x, y)=\delta_{h}(x) \delta_{h}(y) \\
\delta_{h}(x)=\left\{\begin{array}{ll}
\frac{1}{4 h}\left(1+\cos \left(\frac{\pi x}{2 h}\right)\right) & |x| \leq 2 h \\
0 & |x| \geq 2 h
\end{array} .\right.
\end{gathered}
$$

where $h=\Delta x$ for the delta function in the $x$ direction and $h=\Delta y$ for the delta function in the $y$ direction.

Because of the stencil width of $4 h$ in the definition of the delta function in (21), the force at any given immersed boundary point affects only the Eulerian force density at the 16 nearest gridpoints. Because the force at an immersed boundary point contributes to the Eulerian force density over a nonzero area in the discrete equations, this operation is known as the force spreading operation.

Equation (19) is a simple interpolation operation and works much like the spreading operator but in reverse; the interpolated field at the immersed boundary point would be an average of the surrounding values instead of being much bigger than them all.

The Navier-Stokes equations, equations (3) and (4), are solved using a projection method, meaning that (3) is first solved with an approximation to the pressure gradient while ignoring the incompressibility constraint, and then a correction is performed (which involves solving a Poisson equation) in order to enforce the incompressibility constraint and obtain a more accurate pressure approximation. Equations (3) and (4) are discretized with the following discrete analogs of $\nabla, \nabla \cdot \Delta$, and $(\mathbf{u} \cdot \nabla)$ :

$$
\begin{aligned}
\left(\nabla^{h} \cdot \mathbf{u}\right)_{i j} & =\frac{u_{i+1, j}-u_{i-1, j}}{2 \Delta x}+\frac{v_{i, j+1}-v_{i, j-1}}{2 \Delta y} \\
\left(\nabla^{h} p\right)_{i j} & =\left(\frac{p_{i+1, j}-p_{i-1, j}}{2 \Delta x}, \frac{p_{i, j+1}-p_{i, j-1}}{2 \Delta y}\right) \\
\left(\Delta_{\text {wide }}^{h} p\right)_{i j} & =\frac{p_{i+2, j}-2 p_{i, j}+p_{i-2, j}}{4 \Delta x^{2}}+\frac{p_{i, j+2}-2 p_{i, j}+p_{i, j-2}}{4 \Delta y^{2}} \\
\left(\Delta_{\text {tight }}^{h} p\right)_{i j} & =\frac{p_{i+1, j}-2 p_{i, j}+p_{i-1, j}}{\Delta x^{2}}+\frac{p_{i, j+1}-2 p_{i, j}+p_{i, j-1}}{\Delta y^{2}} \\
\left(\left(\mathbf{u} \cdot \nabla^{h}\right) c\right)_{i j} & =u_{i, j}\left(\frac{c_{i+1, j}-c_{i-1, j}}{2 \Delta x}\right)+v_{i, j}\left(\frac{c_{i, j+1}-c_{i, j-1}}{2 \Delta y}\right)
\end{aligned}
$$

Both discrete Laplacian operators are used, with the tight stencil used for the viscous terms and the wide stencil used for the intermediate Poisson solve in the projection (or "correction") step.

As stated in the text when solving the simpler equations for Stokes flow three Poisson solves are performed (one for the pressure, followed by one for each of the velocity components) [40].

The most straightforward discretization of $\mathcal{M}$ is to write the force at an immersed boundary point as a difference in the tensions on either side of that point. Assuming a single closed boundary with no external links, this can be written as

$$
\begin{aligned}
\mathbf{M}_{k}= & \frac{\left(T_{k+1 / 2}(t) \boldsymbol{\tau}_{k+1 / 2}(t)\right)-\left(T_{k-1 / 2}(t) \boldsymbol{\tau}_{k-1 / 2}(t)\right)}{\Delta s} \\
= & \frac{\left(\frac{T_{0}}{\Delta s}\left(\left\|\mathbf{X}_{k+1}(t)-\mathbf{X}_{k}(t)\right\|-\ell_{0}\right) \frac{\mathbf{X}_{k+1}(t)-\mathbf{X}_{k}(t)}{\left\|\mathbf{X}_{k+1}(t)-\mathbf{X}_{k}(t)\right\|}\right)}{\Delta s}- \\
& \frac{\left(\frac{T_{0}}{\Delta s}\left(\left\|\mathbf{X}_{k-1}(t)-\mathbf{X}_{k}(t)\right\|-\ell_{0}\right) \frac{\mathbf{X}_{k-1}(t)-\mathbf{X}_{k}(t)}{\left\|\mathbf{X}_{k-1}(t)-\mathbf{X}_{k}(t)\right\|}\right)}{\Delta s}
\end{aligned}
$$

where $\ell_{0}$ is the resting length of the "springs" connecting Immersed Boundary points. The reason for calling the connection between Immersed Boundary points a "spring" in the discrete set of equations is because of the form of (28): $T_{0} / \Delta s$ serves as a spring constant, $\left\|\mathbf{X}_{i}-\mathbf{X}_{k}\right\|-\ell_{0}$ is the length by which the connection between IB points $i$ and $k$ has been stretched, and $\left(\mathbf{X}_{i}-\mathbf{X}_{k}\right) /\left\|\mathbf{X}_{i}-\mathbf{X}_{k}\right\|$ is a unit vector in the direction of the connection, making this look just like a Hooke's Law spring.

Noting the similarity in the two terms of (28), we can instead write the force as a sum over IB points connected to IB point $k$ :

$$
\mathbf{M}_{k}=\sum_{i} \frac{T_{0}}{\Delta s}\left(\left\|\mathbf{X}_{i}(t)-\mathbf{X}_{k}(t)\right\|-\ell_{0}\right) \frac{1}{\Delta s} \frac{\mathbf{X}_{i}(t)-\mathbf{X}_{k}(t)}{\left\|\mathbf{X}_{i}(t)-\mathbf{X}_{k}(t)\right\|}
$$


An additional advantage of writing in this manner is that it also makes clear how to handle external links connecting objects.

Gathering the temporal and spatial discretizations, the sequence of equations we solve is

$$
\begin{gathered}
\mathbf{M}_{k}^{n}=\sum_{i} \frac{T_{0}}{\Delta s}\left(\left\|\mathbf{X}_{i}^{n}-\mathbf{X}_{k}^{n}\right\|-\ell_{0}\right) \frac{1}{\Delta s} \frac{\mathbf{X}_{i}^{n}-\mathbf{X}_{k}^{n}}{\left\|\mathbf{X}_{i}^{n}-\mathbf{X}_{k}^{n}\right\|} . \\
\mathbf{f}_{i j}^{n}=\sum_{k} \mathbf{F}_{k}^{n} \delta_{h}\left(\mathbf{x}_{i j}-\mathbf{X}_{k}^{n}\right) \Delta s \\
\rho \frac{\mathbf{u}_{i j}^{n+1}-\mathbf{u}_{i j}^{n}}{\Delta t}=-\left(\nabla^{h} p\right)_{i j}^{n+\frac{1}{2}}-\rho\left[\left(\mathbf{u} \cdot \nabla^{h}\right) \mathbf{u}\right]_{i j}^{n+\frac{1}{2}}+\frac{\mu}{2}\left(\Delta^{h}\left(\mathbf{u}^{n+1}+\mathbf{u}^{n}\right)\right)_{i j}+\mathbf{f}_{i j}^{n} \\
\frac{\left(\nabla^{h} \cdot \mathbf{u}^{n+1}\right)_{i j}=0}{\Delta t}=\sum_{i j} \mathbf{u}_{i j}^{n+1} \delta_{h}\left(\mathbf{x}_{i j}-\mathbf{X}_{k}^{n}\right) \Delta x \Delta y .
\end{gathered}
$$

In order to preserve the volume in the cells a correction to the membrane velocity is added to enforce the divergence free condition. At the membrane grid point $i$ let $u_{i}$ denote the velocity, $n_{i}$ be the unit normal vector, $L$ be the arc length of the membrane, and $d s_{i}$ be the arc length between grid point $i$ and $i-1$. The average outward velocity is calculated by

$$
a=\frac{1}{L} \sum u_{i} \cdot n_{i} d s_{i}
$$

The velocity is then adjusted at each point on the membrane by

$$
\bar{u}_{i}=u_{i}-a \bar{n}_{i}
$$

where $\bar{u}_{i}$ is the new velocity and $\overline{n_{i}}$ is a unit vector which is the average of the normal vectors for point $i$ and the points adjacent to it. Due to computational difficulties, membrane points which are bound to the other cell are not included in the average or the correction. The correction is linearly ramped up from the edge of the region on the membrane of cell-cell contact.

[1] B. Geiger, Z. Avnur, G. Rinnerthaler, H. Hinssen, and V. J. Small, J. Cell Biol. 99, 83s (1984).

[2] C. L. Adams, Y. T. Chen, S. J. Smith, and W. J. Nelson, J. Cell Biol. 142, 1105 (1998).

[3] M. F. Krendel and E. M. Bonder, Cell Motility and the Cytoskeleton 43, 296 (1999).

[4] V. Vasioukhin, C. Bauer, M. Yin, and E. Fuchs, Cell 100, 209 (2000).

[5] N. A. Gloushankova, N. A. Alieva, M. F. Krendel, E. M. Bonder, H. H. Feder, J. M. Vasiliev, and I. M. Gelfand, PNAS 94, 879 (1997).

[6] J. S. Ehrlich, M. D. H. Hansen, and W. J. Nelson, Developmental Cell 3, 259 (2002).

[7] Y. Imamura, M. Itoh, Y. Maeno, S. Tsukita, and A. Nagafuchi, J. Cell Biol. 144, 1311 (1999).

[8] F. Drees, S. Pokutta, S. Yamada, W. J. Nelson, and W. I. Weis, Cell 123, 903 (2005).

[9] S. Yamada, S. Pokutta, F. Drees, W. I. Weis, and W. J. Nelson, Cell 123, 889 (2005).

[10] B. R. Stevenson and D. A. Begg, Journal of Cell Science 107, 367 (1994).

[11] B. Angres, A. Barth, and W. J. Nelson, J. Cell Biol. 134, 549 (1996).

[12] E. M. Kovacs, M. Goodwin, R. G. Ali, A. D. Paterson, and A. S. Yap, Current Biology 12, 379 (2002).

[13] F. M. Helwani, E. M. Kovacs, A. D. Paterson, S. Verma, R. G. Ali, A. S. Fanning, S. A. Weed, and A. S. Yap, J. Cell Biol. 164, 899 (2004).

[14] S. Verma, A. M. Shewan, J. A. Scott, F. M. Helwani, N. R. den Elzen, H. Miki, T. Takenawa, and A. S. Yap, Journal of Biological Chemistry 279, 34062 (2004).

[15] J. A. Scott, A. M. Shewan, N. R. den Elzen, J. J. Loureiro, F. B. Gertler, and A. S. Yap, Molecular Biology of the Cell 17, 1085 (2006).

[16] M. D. Hansen and M. C. Beckerle, Journal of Biological Chemistry 281, 16178 (2006).

[17] C. S. Peskin, J. Comp. Phys. 25, 220 (1977).

[18] S. Jadhav, Y. C. Kit, K. Konstantopoulos, and C. D. Eggleton, J. Biomech. 40, 2891 (2007).

[19] K. A. Rejniak, J. Theor. Biol. 247, 186 (2007).

[20] D. C. Bottino, J. Comp. Phys. 147, 86 (1998).

[21] D. Boal, Mechanics of the Cell (Cambridge Unversity Press, 2001). 
[22] C. S. Peskin and D. M. McQueen, Journal of Compuatational Physics 81, 372 (1989).

[23] C. T. Lim, M. Dao, S. Suresh, C. H. Sow, and K. T. Chew, Acta Mater. 52, 1837 (2004).

[24] M. Dao, C. T. Lim, and S. Suresh, J. Mech. Phys. Solids 51, 2259 (2003).

[25] Y. Rabinovich, M. Esayanur, S. Daosukho, K. Byer, H. El-Shall, and S. Khan, J. Colloid Interface Sci. 285, 125 (2005).

[26] J. H. Hoh and C.-A. Schoenenberger, J. Cell Sci. 107, 1105 (1994).

[27] E. Newren, Ph.D. thesis, University of Utah (2007).

[28] Y. Sako, A. Nagafuchi, S. Tsukita, M. Takeichi, and A. Kusumi, J. Cell Biol. 140, 1227 (1998).

[29] W. Baumgartner, P. Hinterdorfer, W. Ness, A. Raab, D. Vestweber, H. Schindler, and D. Drenckhahn, Proc. Nat. Acad. Sci. USA 97, 4005 (2000).

[30] Y. C. Fung, Biomechanics: Mechanical Properties of Living Tissues (Springer-Verlag, 1993).

[31] E. Evans and A. Yeung, Biophys J 56, 151 (1989).

[32] W. S. Rasband, Imagej, U.S. National Institutes of Health, Bethesda, Maryland, USA (1997-2007), http://rsb.info.nih.gov/ij/.

[33] B. Vasiev and C. Weijer, J. Theor. Biol. 223, 347 (2003).

[34] J. C. Dallon and H. G. Othmer, J. Theor. Biol. 231, 203 (2004).

[35] D. Drasdo, Single-Cell-Based Models in Biolgy and Medicine (Birhauser, 2007), chap. Center-based Single-cell Models: An Approach to Multi-cellular Organization Based on a Conceptual Analogy to Colloidal Particles.

[36] M. Prass, K. Jacobson, A. Mogilner, and M. Radmacher, J Cell Biol. 174, 767 (2006).

[37] A. C. L, W. J. Nelson, and S. S. J., J. of Cell Biol. 135, 1899 (1996).

[38] M. Yoshigi, L. M. Hoffman, C. C. Jensen, H. J. Yost, and M. C. Beckerle, J. Cell Biol. 171, 209 (2005).

[39] C. S. Peskin, Journal of Computational Physics 25, 220 (1977).

[40] R. J. Leveque and Z. Li, SIAM J Sci. Comput. 18, 709 (1997). 